\title{
Préparation du Canada en cas de grippe pandémique : Guide de planification pour le secteur de la santé
}

\author{
B Henry ${ }^{1,2}$ au nom du Groupe de travail sur la préparation du Canada en cas de grippe \\ pandémique (GTPCP)
}

\section{Résumé}

La préparation en cas de pandémie nécessite une approche à multiples facettes dans le cadre de laquelle tous les ordres de gouvernement collaborent. Le guide Préparation du Canada en cas de grippe pandémique : Guide de planification pour le secteur de la santé (PCGP) est un document d'orientation qui présente les activités de préparation clés du secteur de la santé conçues pour garantir que le Canada est prêt à réagir à la prochaine pandémie de grippe. Le présent article esquisse l'approche canadienne pour la préparation à la grippe pandémique décrite dans le document maître du PCGP. Au Canada, la planification pour la préparation à la grippe pandémique se fait à l'intérieur d'un réseau d'exigences législatives et de cadres d'urgence aux échelons fédéral, provincial, territorial et international. Le plan contient plusieurs principes directeurs, notamment la collaboration entre les gouvernements et les intervenants, la prise de décisions fondées sur des données probantes, la proportionnalité et la souplesse de l'intervention pour qu'elle soit adaptée à la situation, l'adoption d'une approche préventive, le recours aux pratiques et systèmes établis, ainsi que l'intégration explicite des principes éthiques dans tous les processus décisionnels et toutes les décisions. On y énonce les rôles et responsabilités des gouvernements fédéral, provinciaux et territoriaux, et trois outils de planification sont fournis : des hypothèses de planification fondées sur des preuves; de multiples scénarios pour soutenir le processus décisionnel; et des termes descriptifs tels que le début, le pic et la fin d'une vague pandémique, plutôt que la terminologie des phases, pour désigner les éléments déclencheurs de l'intervention. De façon générale, le document maître du PCGP présente une approche adaptable et coordonnée de gestion du risque en cas de pandémie de grippe. Il s'agit d'un document évolutif qui sera régulièrement mis à jour.
Affiliation

${ }^{1}$ Présidente du GTPCP

${ }^{2}$ Bureau de l'administrateur provincial de la santé, Victoria (Colombie-Britannique)

*Correspondance : CPIPTGSecretariat-GTPCPSecreta riat@phac-aspc.gc.ca

Citation proposée : B. Henry au nom du Groupe de travail sur la préparation du Canada en cas de grippe pandémique (GTPCGP). Préparation du Canada en cas de grippe pandémique : Guide de planification pour le secteur de la santé. Relevé des maladies transmissibles au Canada. 2018;44(1):7-10.

https://doi.org/10.14745/ccdr.v44i01a02f

\section{Introduction}

Les pandémies de grippe sont des événements peu fréquents qui surviennent lorsqu'un nouveau virus de la grippe $A$ se transmettant de manière soutenue d'une personne à l'autre entraîne une épidémie généralisée de maladie chez les humains. Les gouvernements doivent se préparer à l'avance à intervenir en cas de pandémie de grippe, puisqu'il est impossible de prévoir à quel moment une pandémie surviendra ni quelle en sera la gravité. Cet article présente un résumé du document maître de l'approche de planification du Canada en cas de pandémie de grippe, telle qu'elle est décrite dans le document Préparation du Canada en cas de grippe pandémique : Guide de planification pour le secteur de la santé (PCGP) (1). Le PCGP fournit une orientation aux autorités fédérales, provinciales et territoriales (FPT) responsables de la préparation et de l'intervention en cas de pandémie de grippe; il cible en premier lieu les ministères FPT de la Santé et les autres ministères ayant des responsabilités en matière de santé. Il ne s'agit pas en soi d'un plan d'intervention en cas de pandémie.
Tous les gouvernements FPT doivent collaborer efficacement à la planification et à la prestation des activités d'intervention. Ainsi, le PCGP explique comment les autorités FPT «travailleront de concert pour assurer une approche coordonnée et uniforme à l'échelle du secteur de la santé en ce qui concerne la préparation et les interventions en cas de pandémie » (1). La stratégie et l'orientation fournies dans le document maître du PCGP ont été approuvées par le Conseil du Réseau pancanadien de santé publique (RSP) (2) et la Conférence des sousministres FPT de la Santé, et l'on prévoit que la planification par les autorités FPT s'harmonisera à l'orientation stratégique du PCGP.

Puisque le PCGP se fonde autant que possible sur les fonctions et structures existantes dans le secteur de la santé, comme les mesures de surveillance et de contrôle, il peut appuyer les plans $d^{\prime}$ intervention tous risques applicables à n'importe quel type d'urgence de santé publique. 


\section{Le plan actualisé}

Le PCGP est un guide de planification à l'intention du secteur de la santé pour la préparation et l'intervention en cas de grippe pancanadienne. II vise à réduire au minimum la morbidité et la mortalité en général, de même qu'à atténuer les perturbations sociales que pourrait causer une pandémie de grippe. Parmi les nouveaux aspects abordés dans le PCGP, mentionnons :

- Des approches et des principes directeurs, comme la prise en considération de facteurs éthiques et de la diversité du Canada;

- L'adoption d'une approche de gestion du risque, accompagnée d'hypothèses de planification mises à jour;

- Des outils de planification pour aider les provinces et les territoires (PT) à élaborer leurs propres plans.

Certains éléments de la version actualisée du PCGP reflètent les leçons retenues à la suite de la pandémie de grippe H1N1 de 2009 (3). Par exemple, compte tenu de la demande élevée pour certains éléments de l'intervention, comme les activités de surveillance et le matériel médical pour les soins intensifs, on a recommandé de renforcer les systèmes de surveillance et la capacité épidémiologique, ainsi que les liens avec les fournisseurs de soins de première ligne. En réponse aux variations quant au moment et à l'intensité des vagues pandémiques, on a prévu une plus grande adaptabilité et extensibilité des mesures d'intervention, avec un ensemble d'éléments déclencheurs déterminant les conditions pandémiques qui devraient entraîner l'activation ou la désactivation de certaines interventions.

Le document maître décrit le contexte et les justifications qui sous-tendent la planification de la préparation en cas de pandémie de grippe en général, de même que l'approche adoptée dans le contexte canadien en particulier. Cette orientation stratégique générale est accompagnée d'une série d'annexes techniques qui fournissent des directives et des conseils plus détaillés pour bon nombre des éléments fonctionnels clés de la préparation et de l'intervention en cas de pandémie, tout en incorporant les principes stratégiques plus généraux du document maître. À la lumière des leçons retenues de la pandémie de grippe H1N1 de 2009, on a mis à jour récemment les annexes pour trois fonctions d'intervention clés : la surveillance, les services en laboratoire et la vaccination.

Le présent article est le deuxième d'une série d'articles faisant le point sur les différentes sections du PCGP. D'autres articles sur les annexes portant sur la stratégie de laboratoire et la stratégie de surveillance sont également présentés plus loin dans ce numéro du Relevé des maladies transmissibles au Canada (RMTC) $(4,5)$. Le premier article de la série présentait un résumé de l'Annexe traitant de la vaccination du PCGP (6).

\section{Contexte de la planification}

\section{Législation et cadres d'urgence}

Au Canada, la planification pour la préparation à la grippe pandémique se fait à l'intérieur d'un réseau d'exigences législatives et de cadres d'urgence aux échelons fédéral, provincial, territorial et international. Les plans de préparation du gouvernement fédéral pour les urgences de santé publique font partie du système général de gestion des urgences, qui est géré par Sécurité publique Canada (7), et il existe également un système de plans d'urgence sanitaire FPT. Le Canada collabore également avec plusieurs partenaires internationaux pour garantir la préparation régionale en cas de pandémie de grippe, en vertu d'accords tels que le Cadre de préparation en cas de grippe pandémique (Assemblée mondiale de la Santé) (8) et le Plan nord-américain contre l'influenza animale et la pandémie d'influenza ${ }^{(9)}$, avec le Mexique et les États-Unis.

\section{Autres facteurs à considérer pour la planification}

La version actualisée du PCGP a été préparée en tenant compte de la diversité du Canada, qui est le reflet de la variabilité et de la taille géographique du pays, de même que de la diversité ethnique, linguistique, religieuse et culturelle de sa population, en plus des différents modes de vie adoptés. Les facteurs à considérer dans la planification incluent par exemple la présence, d'un bout à l'autre du pays, de nombreux villages éloignés et isolés qui sont moins bien servis par les services de santé et autres services, ainsi que les personnes et les groupes qui sont plus vulnérables aux urgences sanitaires, comme les personnes ayant une déficience physique ou mentale, les gens à faible revenu ou les sans-abris.

Ces facteurs sont particulièrement pertinents dans le contexte de la préparation et de l'intervention en cas de pandémie, principalement parce qu'ils nécessitent une intervention adaptable aux conditions locales et aux besoins de certaines personnes ou de groupes vulnérables. Les principes éthiques sont présentés de façon explicite dans la version actualisée du PCGP; ils orientent les décisions de manière à ce qu'elles soient fondées sur la santé et les intérêts d'une population, plutôt que de s'appuyer sur des principes éthiques cliniques fondés sur les intérêts des individus. Cette orientation suppose la nécessité d'encourager un sens de la solidarité au sein d'une communauté, de même qu'un sens de la réciprocité à l'endroit de ceux qui pourraient avoir besoin d'un soutien accru.

\section{Principes directeurs}

Le PCGP actualisé s'appuie sur un ensemble de principes directeurs, comme la collaboration entre les gouvernements et les intervenants, la prise de décisions fondées sur des données probantes, ainsi que la proportionnalité et la souplesse de I'intervention pour qu'elle soit adaptée à la situation. On applique aussi trois approches plus générales : l'adoption d'une approche préventive ou protectrice, surtout aux stades précoces, où les incertitudes sont les plus grandes; le recours aux pratiques et systèmes établis, plutôt que d'essayer d'adopter de nouvelles approches pendant une urgence; ainsi que l'intégration explicite des principes éthiques dans tous les processus décisionnels et toutes les décisions.

\section{Directives pour la préparation et I'intervention}

\section{Coordination des rôles et responsabilités}

Pour la préparation et l'intervention en cas de pandémie de grippe, il faut adopter une approche pangouvernementale visant à garantir que toutes les ressources nécessaires sont mobilisées de façon à réduire les répercussions sur la santé, la société et l'économie, et ces contributions doivent être coordonnées. Les activités du secteur de la santé pour la préparation en cas de pandémie qui sont décrites dans le PCGP exigent la participation des gouvernements FPT et des gouvernements d'autres pays. En 
outre, bon nombre des fonctions opérationnelles sont assumées par tout un éventail de disciplines professionnelles œuvrant à l'intérieur et à l'extérieur du secteur de la santé, comme les professionnels de la santé, les responsables internationaux de la réglementation, les fabricants de vaccins et les organisations non gouvernementales. La délimitation des responsabilités des gouvernements FPT relativement à ces fonctions, de même que les mécanismes régissant leur collaboration, sont des aspects majeurs des activités de préparation décrites dans le PCGP.

À l'échelle internationale, l'Organisation mondiale de la Santé (OMS) effectue des évaluations du risque à l'échelle mondiale, déclare les urgences de santé publique qui sont préoccupantes sur la scène internationale, sélectionne les souches pour les vaccins antipandémiques et détermine à quel moment il faut changer la production des vaccins pour la grippe saisonnière pour des vaccins antipandémiques. La liaison avec l'OMS et les autres organisations internationales pour la gestion d'une pandémie relève de la responsabilité du gouvernement fédéral.

La coordination d'une intervention pancanadienne nécessite des infrastructures collectives et des activités coordonnées. Par exemple, le gouvernement fédéral est responsable des volets réglementaires liés à la mise à l'essai et à l'approbation des vaccins contre la grippe et des médicaments antiviraux, des négociations avec les fabricants et de la passation de marchés pour les achats par les autorités FPT des vaccins antigrippaux et des médicaments antiviraux, de l'entretien et de la mobilisation des fournitures médicales dans la Réserve nationale stratégique d'urgence, ainsi que de l'acquisition de fournitures supplémentaires. Les PT sont responsables de l'achat, de la distribution et de l'administration des vaccins et des antiviraux au sein de leur territoire de compétence.

\section{Approche de la gestion du risque}

La version actualisée du PCGP propose une approche de la gestion du risque pour la prise de décisions afin de gérer les incertitudes qui sont inhérentes à la planification de la préparation en cas de pandémie de grippe. La gestion du risque est une approche systématique permettant d'établir le meilleur plan d'action possible dans un environnement incertain, en déterminant, en évaluant et en communiquant les risques et en prenant les mesures qui s'imposent. Cette approche est soutenue par les principes du PCGP que sont la prise de décisions fondées sur des données probantes, la proportionnalité et la souplesse, ainsi que l'approche préventive ou protectrice dans des conditions incertaines.

\section{Outils pour la planification de la préparation en cas de pandémie}

Vu les nombreuses variables possibles de la planification en cas de pandémie grippale, il est difficile de procéder à une gestion complète du risque. Le PCGP actualisé contient trois grands outils de planification : les hypothèses de planification, les scénarios de planification en cas de pandémie, ainsi que les phases de la pandémie et les éléments déclencheurs d'intervention.

Les hypothèses de planification sont des énoncés hypothétiques fondés sur des preuves; elles servent à orienter la gestion des incertitudes et forment un cadre utile pour les phases de planification. À mesure que la pandémie évoluera, les données probantes émergentes remplaceront ces hypothèses pour orienter l'intervention.
On a avancé de multiples scénarios afin d'aider à cerner les risques et ainsi soutenir la planification et la prise de décisions fondées sur des données probantes. Les scénarios de planification fournissent un point de départ pour aborder les implications et les risques d'une pandémie en fonction de ses répercussions sur la population, qui vont de faibles à élevées.

Le PCGP définit les termes descriptifs employés dans les phases de planification, comme le début, le pic et la fin d'une vague pandémique. Auparavant, on employait la terminologie des phases de l'OMS (phase interpandémique, phase d'alerte, phase pandémique et phase de transition) pour décrire l'activité pandémique au pays ou au sein d'un territoire de compétence. Les éléments déclencheurs d'intervention constituent un guide pour lancer, modifier ou interrompre les activités FPT. L'intervention en cas de pandémie devrait être adaptée à la situation locale, de façon à garantir que le niveau d'intervention à l'échelon provincial, régional ou local est adéquat en fonction de la situation.

\section{Mesure et évaluation}

La préparation et l'intervention en cas de pandémie s'inscrivent dans une démarche complexe qui exige les efforts coordonnés de tous les ordres de gouvernement, en collaboration avec les intervenants. Pour assurer l'exhaustivité et l'efficacité des plans de lutte contre la pandémie (ou les plans tous risques, selon le territoire de compétence), les territoires de compétence devraient mesurer leur état de préparation, tester leurs plans régulièrement et évaluer leur intervention en cas de pandémie.

\section{Discussion}

Le PCGP actualisé répond à plusieurs défis inhérents à la planification de la préparation et de l'intervention en cas de pandémie de grippe au Canada, incluant non seulement les incertitudes intrinsèques des pandémies de grippes, mais aussi l'étendue, la diversité et les différents secteurs de compétence au Canada.

Le PCGP aborde les incertitudes associées à la grippe pandémique au moyen d'une approche de gestion du risque adaptable en fonction du niveau de répercussions de la pandémie (faibles, modérées ou élevées) et de l'évolution de ces répercussions à mesure que la pandémie progresse. Cette approche offre aussi la souplesse dont les décideurs ont besoin pour adapter l'intervention aux besoins et aux capacités des différentes régions du Canada, en s'ajustant aux variations régionales quant au moment et à l'intensité des répercussions de la pandémie, ainsi qu'aux diverses communautés et populations.

S'il est nécessaire d'offrir une certaine souplesse pour permettre aux différents territoires de compétence d'adapter leurs activités de planification et d'intervention en cas de pandémie aux conditions et aux besoins des régions, il faut aussi avoir des objectifs communs et une approche uniforme pour permettre aux territoires de compétence de collaborer à la réalisation des activités d'intervention. Afin d'assurer l'uniformité de l'approche de planification en cas de pandémie parmi les différents territoires de compétence FPT et pour faciliter la collaboration entre les partenaires de l'intervention, le PCGP énonce une série de principes et prend en considération les enjeux éthiques, la diversité de la population et les groupes vulnérables au Canada, dont doivent tenir compte les plans de lutte contre la pandémie. Sur le plan opérationnel, il est essentiel que les territoires de compétence collaborent entre eux et en leur sein pour assurer 
une intervention efficace en cas de pandémie. Le PCGP délimite les rôles et responsabilités pour les activités de préparation et d'intervention à l'échelle nationale et établit un processus pour les interactions et la communication entre les territoires de compétence et en leur sein. Ces rôles, structures et processus collaboratifs sont un aspect important de la préparation en cas de pandémie au Canada.

Les principes généraux, les facteurs à considérer, l'approche de gestion du risque, les structures et les processus de collaboration énoncés dans le PCGP sont repris dans les documents d'orientation plus détaillés qui ont été préparés pour chacun des éléments de l'intervention.

\section{Conclusion}

Le PCGP est un document évolutif qui sera régulièrement mis à jour, selon les besoins, afin d'y intégrer les nouveautés en matière d'informations, de changements ou d'accords législatifs et de pratiques exemplaires. Un examen plus exhaustif du PCGP et de ses annexes sera effectué tous les cinq ans afin de s'assurer que le document est à jour et qu'il répond aux besoins des gouvernements FPT, des professionnels de la santé et des intervenants. Le prochain examen complet du document maître aura lieu en 2019.

\section{Déclaration des auteurs}

Groupe de travail sur la préparation du Canada en cas de grippe pandémique (GTPCP) : Henry B. (présidente), Alfieri A., Gant S., Gemmill I.,Hatchette T., Jayaraman G., Schwartz B.

Secrétariat du GTPCP : Paddle L., Stirling R., Gadient S.

ASPC : Charos G., Williams J.

\section{Conflit d'intérêt}

Aucun.

\section{Remerciements}

Merci à Anne Wiles, qui a préparé la première ébauche de ce résumé.

\section{Financement}

Les travaux du Groupe de travail sur la préparation du Canada en cas de grippe pandémique sont financés par l'Agence de la santé publique du Canada.

\section{Références}

1. Agence de la santé publique du Canada. Préparation du Canada en cas de grippe pandémique : Guide de planification pour le secteur de la santé. Ottawa (ON): PHAC; 2015. https://www.canada.ca/fr/sante-publique/ services/grippe-influenza/preparation-canada-cas-grippepandemique-guide-planification-secteur-sante.html

2. Réseau pancanadien de santé publique. http://www.phn-rsp. ca/index-fra.php

3. Agence de la santé publique du Canada et Santé Canada. Leçons à retirer : Réponse de l'Agence de la santé publique du Canada et de Santé Canada à la pandémie de grippe H1N1 de 2009. Ottawa (ON): ASPC; 2010. http://www. phac-aspc.gc.ca/about_apropos/evaluation/reportsrapports/2010-2011/h1n1/pdf/h1n1-fra.pdf

4. Henry B au nom du Groupe de travail sur la préparation du Canada en cas de grippe pandémique. Préparation du Canada en cas de grippe pandémique : Stratégie de laboratoire. Relevé des maladies transmissible au Canada. 2018;44(1):11-5. https://www.canada.ca/fr/santepublique/services/rapports-publications/releve-maladiestransmissibles-canada-rmtc/numero-mensuel/2018-44/rmtcvolume-44-1-4-janvier-2018/preparation-canada-cas-grippepandemique-strategie-laboratoire.html

5. Henry B au nom du Groupe de travail sur la préparation du Canada en cas de grippe pandémique. Préparation du Canada en cas de grippe pandémique : Stratégie de surveillance. Relevé des maladies transmissible au Canada. 2018;44(1):16-20. https://www.canada.ca/fr/santepublique/services/rapports-publications/releve-maladiestransmissibles-canada-rmtc/numero-mensuel/2018-44/rmtcvolume-44-1-4-janvier-2018/preparation-canada-cas-grippepandemique-strategie-surveillance.html

6. Henry B, Gadient S au nom du Groupe de travail sur la préparation du Canada en cas de grippe pandémique. Stratégie canadienne de vaccination en cas de pandémie. Relevé des maladies transmissibles au Canada.

2017;43(7/8):180-4. https://www.canada.ca/content/dam/ phac-aspc/migration/phac-aspc/publicat/ccdr-rmtc/17vol43/ dr-rm43-7-8/assets/pdf/17vol43_7_8-ar-05-fra.pdf

7. Sécurité publique Canada. Gestion des urgences. 2016. https://www.securitepublique.gc.ca/cnt/mrgnc-mngmnt/ index-fr.aspx

8. World Health Organization. World Health Assembly Pandemic Influenza Preparedness Framework. 2011 World Health Organization. http://www.who.int/influenza/pip/en/

9. Sécurité publique Canada. Plan nord-américain contre I'influenza animale et la pandémie d'influenza. Ottawa (ON): Public Safety Canada; 2016. https://www.securitepublique. gc.ca/cnt/rsrcs/pblctns/nml-pndmc-nflnz/index-fr.aspx

10. Agence de la santé publique du Canada. Réserve nationale stratégique d'urgence. Ottawa (ON): ASPC; 2015. https:// www.canada.ca/fr/sante-publique/services/mesuresinterventions-urgence/reserve-nationale-strategiqueurgence.html 